\title{
Diastereo- and enantioselective syntheses of heteroaryloxiranes
}

\author{
Luigino Troisi, ${ }^{* a}$ Luisella De Vitis, ${ }^{a}$ Catia Granito, ${ }^{a}$ Pierangelo Metrangolo, ${ }^{b}$ \\ Tullio Pilati, ${ }^{c}$ and Ludovico Ronzini ${ }^{a}$
}

${ }^{a}$ Dipartimento di Scienze e Tecnologie Biologiche ed Ambientali, University of Lecce, Via Prov.le Lecce-Monteroni, I-73100 Lecce, Italy

${ }^{b}$ Dipartimento di Chimica, Materiali ed Ingegneria Chimica "G. Natta”, Politecnico di Milano, Via Mancinelli 7, I-20131 Milan, Italy

c CNR “Institute of Molecular Science and Technology”, University of Milan, Via C. Golgi 19, I-20133 Milan, Italy

E-mail: luigino.troisi@unile.it

(received 05 Feb 04; accepted 18 Aug 04; published on the web 30 Aug 04)

\begin{abstract}
$\alpha$-Chloroheteroarylalkyllithiums generated by deprotonation of 2-(1-chloroalkyl)-heterocycles with $n$-BuLi in THF at $-78{ }^{\circ} \mathrm{C}$, were found to react as Darzens-type reagents with racemic and optically pure 2- and 3-methylcyclohexanones. Heteroaryloxiranes containing new stereocentres were isolated in a diastereo- and enantioselective manner.
\end{abstract}

Keywords: $\alpha$-Chloroheteroarylalkyllithium, methylcyclohexanone, heteroaryloxirane

\section{Introduction}

The deprotonation of $\alpha$-chloroheteroarylalkanes with $n$-butyllithium ( $n$-BuLi) or lithium diisopropylamide (LDA) at $-78{ }^{\circ} \mathrm{C}$ in THF, provides carbanions relatively stable due to the presence, in their framework, of an aza heterocycle in $\alpha$ position and an adjacent electronegative chlorine atom, which add a further electron-withdrawing effect. ${ }^{1}$ An interesting diastereoselectivity has been observed in the coupling reaction of the above carbanions with carbonyl or iminic compounds affording oxiranes and aziridines, respectively. ${ }^{2}$ The option of deblocking the oxiranyl ring $^{3,4}$ as well as the potential elaboration of the aza heterocycle to a carbonylic function, make these compounds extremely useful intermediates in the organic synthesis of biologically active compounds. ${ }^{5}$ It has been reported that the coupling reaction of lithium azaenolates of chiral oxazolines with carbonyl compounds ${ }^{6}$ produces asymmetric induction leading to chiral oxiranes. Moreover, the deprotonation of substituted oxazolinyloxiranes with strong bases has been reported, affording oxiranyl anions chemically and configurationally stable to react stereospecifically with electrophiles; ${ }^{7}$ recently the coupling 
of trisubstituted oxazolinyloxiranes with electrophiles has been described to give the tetrasubstituted derivatives with complete retention of configuration at the $\beta$-carbon. ${ }^{8}$

Asymmetric induction has been noticed also in the coupling reaction of non chiral lithiated ( $\alpha$-chloroalkyl)heterocycles with various enantiopure imines affording 'one-pot' chiral heterosubstituted aziridines in a diastereoselective manner. ${ }^{9}$ Encouraged by these findings, we decided to investigate a possible diastereo- and enantioselectivity in the coupling of non-chiral heteroarylcarbanions with carbonyl compounds containing stereocentres.

\section{Results and Discussion}

Several $\alpha$-chloroheteroarylalkyllithiums 1-7, prepared as described in the Experimental Section, were added in THF at $-78{ }^{\circ} \mathrm{C}$ of 2 - and 3-methylcyclohexanone, in the racemic and enantiomerically pure form, to give heteroaryloxiranes 1a,b,c,d-14a,b,c,d (Scheme 1).

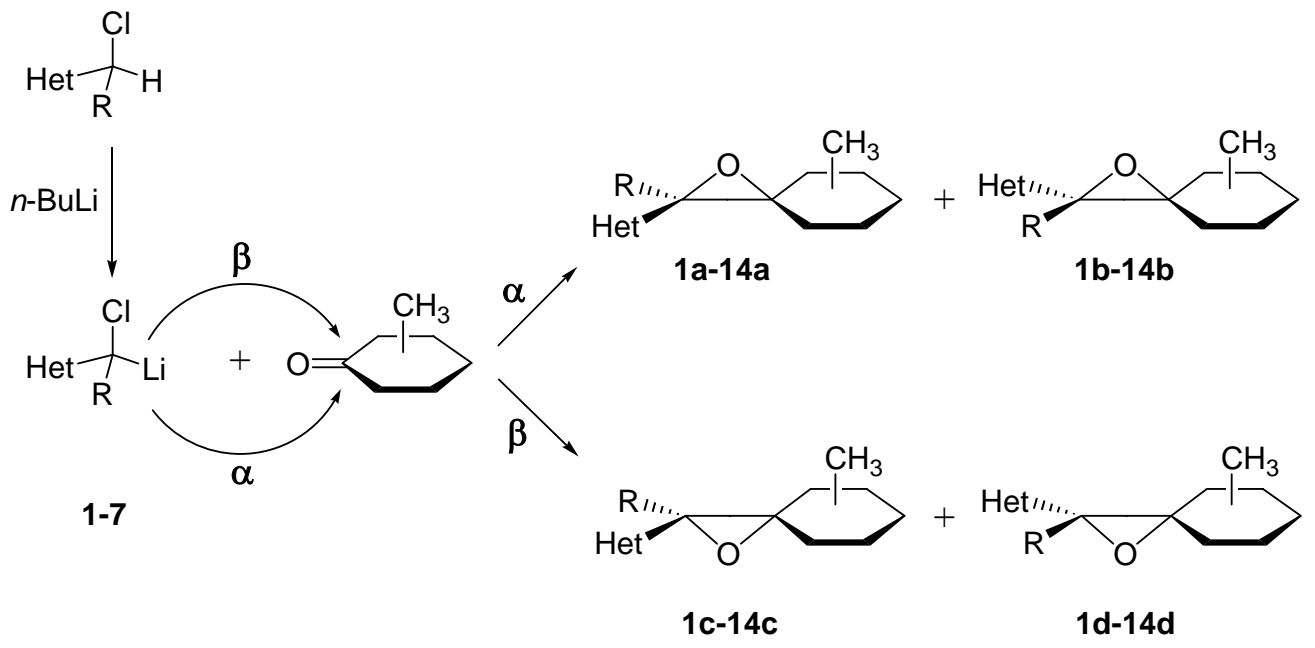

Scheme 1. Coupling reaction of $\alpha$-chloroheteroarylalkyllithiums with 2 - and 3 methylcyclohexanone.

The coupling reaction can occur by two possible attacks of the carbanion to the electrophile affording four possible diastereoisomers. According to the route $\boldsymbol{\alpha}$, a carbanion attack of anti type towards the cyclohexanic $\mathrm{CH}_{3}$ should take place leading to the diastereomers a (trans configuration) and $\mathbf{b}$ (cis configuration). An attack of syn type, as showen by the route $\boldsymbol{\beta}$, should produce diastereoisomers $\mathbf{c}$ (trans configuration) and $\mathbf{d}$ (cis configuration).

The coupling reaction of $\alpha$-chloroheteroarylalkyllithiums 1-7 with 3-methylcyclohexanone in the racemic form (entries 1-7, Table 1) gave products 1a-d, 3a-d, 5a-d, 7a-d, 9a-d, 11a-d, and 13a-d in good yields (50-95\%). Products 2a-d; 4a-d; 6a-d; 8a-d; 10a-d; 12a-d; 14a-d were obtained by analogous reaction of 1-7 with 2-methylcyclohexanone in the racemic form, yields $70-95 \%$ (entries $8-14$, Table 1 ). 
Table 1. Summary of coupling reactions between $\alpha$-chloroheteroarylalkyllithiums and carbonyl compounds affording heteroaryloxiranes

\begin{tabular}{|c|c|c|c|c|c|c|c|}
\hline Entry & & $\begin{array}{l}\text { bstrate } \\
\text { Het }\end{array}$ & $\mathrm{R}$ & Electrophile & $\begin{array}{l}\text { Total } \\
\text { yields }^{[\mathrm{a}]}\end{array}$ & Products & $\mathrm{dr}^{[\mathrm{b}]}=\mathrm{a}: \mathrm{b}: \mathrm{c}: \mathrm{d}$ \\
\hline 1 & 1 & & $\mathrm{R}=\mathrm{H}$ & $\begin{array}{l}\text { 3-methyl- } \\
\text { cyclohexanone }\end{array}$ & 95 & $1 \mathbf{a}-\mathbf{d}$ & 46:traces:46:traces \\
\hline 2 & 2 & “ & $\mathrm{R}=\mathrm{CH}_{3}$ & “ & 65 & $3 \mathbf{a}-\mathbf{d}$ & $50: 0: 50: 0$ \\
\hline 3 & 3 & $\mathrm{H}_{3} \mathrm{C}$ & $\mathrm{R}=\mathrm{H}$ & ، & 50 & $5 a-d$ & $25: 25: 25: 25$ \\
\hline 4 & 4 & ، & $\mathrm{R}=\mathrm{CH}_{3}$ & “ & 90 & $7 \mathbf{a}-\mathbf{d}$ & $43: 7: 43: 7$ \\
\hline 5 & 5 & “6 & $\mathrm{R}=\mathrm{Ph}$ & “ & 65 & $9 a-d$ & $50: 0: 50: 0$ \\
\hline 6 & 6 & $\mathrm{H}_{3} \mathrm{C}$ & $\mathrm{R}=\mathrm{H}$ & “" & 80 & $11 a-d$ & $25: 25: 25: 25$ \\
\hline 7 & 7 & “ & $\mathrm{R}=\mathrm{CH}_{3}$ & “ & 65 & $13 a-d$ & 50:traces:40:traces \\
\hline 8 & 1 & & $\mathrm{R}=\mathrm{H}$ & $\begin{array}{l}\text { 2-methyl- } \\
\text { cyclohexanone }\end{array}$ & 95 & $2 a-d$ & 100:0:0:0 \\
\hline 9 & 2 & “ & $\mathrm{R}=\mathrm{CH}_{3}$ & “ & 90 & $4 a-d$ & $80: 20: 0: 0$ \\
\hline 10 & 3 & $\mathrm{H}_{3} \mathrm{C}^{\prime}$ & $\mathrm{R}=\mathrm{H}$ & ، & 95 & $6 a-d$ & $67: 33: 0: 0$ \\
\hline 11 & 4 & “ & $\mathrm{R}=\mathrm{CH}_{3}$ & “ & 70 & $8 \mathbf{a}-\mathbf{d}$ & $75: 25: 0: 0$ \\
\hline 12 & 5 & “ & $\mathrm{R}=\mathrm{Ph}$ & “ & 72 & $10 a-d$ & $50: 35: 15: 0$ \\
\hline 13 & 6 & $\begin{array}{c}\mathrm{H}_{3} \mathrm{C}- \\
\mathrm{H}_{3} \mathrm{C}\end{array}$ & $\mathrm{R}=\mathrm{H}$ & “6 & 80 & $12 a-d$ & $38: 38: 12: 12$ \\
\hline 14 & 7 & “ & $\mathrm{R}=\mathrm{CH}_{3}$ & “ & 80 & $14 a-d$ & $70: 30: 0: 0$ \\
\hline
\end{tabular}

${ }^{[\mathrm{a}]}$ Isolated yields. ${ }^{[\mathrm{b}]}$ Diastereomeric ratio (dr) determined by GC and ${ }^{1} \mathrm{H}$ NMR spectroscopy.

The results reported in Table 1 could be explained by the following considerations. When 3methylcyclohexanone is used as electrophile (Scheme 1) the steric hindrance exerted by the cyclohexanic $\mathrm{CH}_{3}$ is not significant, consequently the carbanion's attack seems to take place on either of the faces (route $\alpha$ and $\beta$ ) with equal probability. The diastereomeric ratios measured (Table 1, entries 1-7) therefore, showed a predominance of a and $\mathbf{c}$ structures generated by an anti and a syn attack of the $\alpha$-chloroheteroarylalkyllithiums to the 3-methylcyclohexanone, respectively. As these structures are both of trans configuration, we presume that the stereoselective determining step is a transition state energetically favoured, which evolves to the final oxiranic structure. The Scheme 2 shows, as previously reported, ${ }^{10}$ that the stable azaenolate reacts with the ketone affording two possible transition states $\mathbf{T} \mathbf{S}_{\mathbf{1}}$ and $\mathbf{T S}_{\mathbf{2}}$. The probable internal coordination of lithium by the nitrogen and the oxygen atoms generates $\mathbf{T S}_{\mathbf{1}}$ energetically more favoured than $\mathbf{T S}_{\mathbf{2}}$ because of the different interaction between the heterocyclic moiety and the methyl-substituted branch of the ketone. 

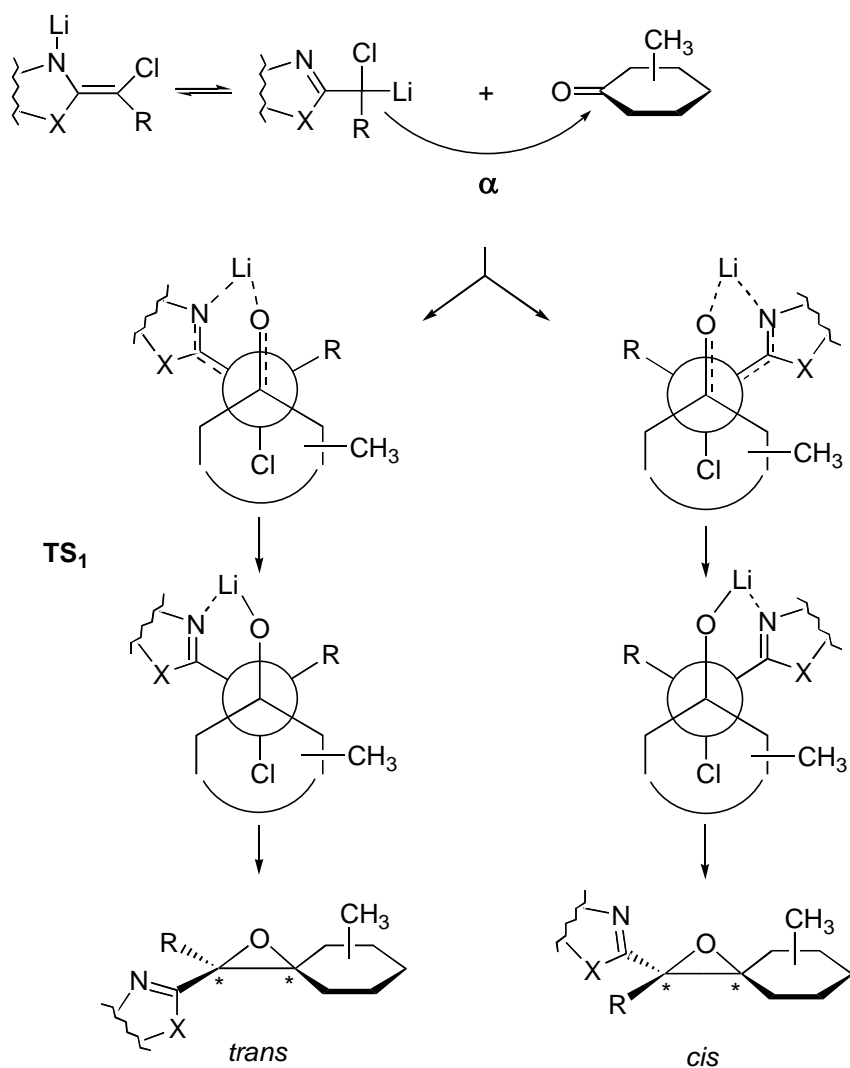

$\mathrm{TS}_{2}$

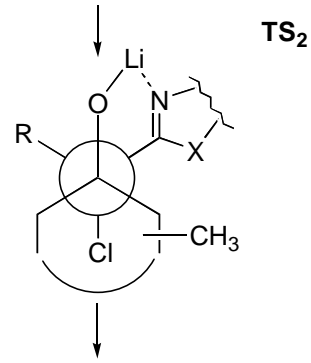

Scheme 2. Suggested transition states explaining the diasteroselectivity of heteroaryloxiranes.

These considerations might explain the trans diastereoselection of $\mathbf{a}$ and $\mathbf{c}$ structures compared with the cis diastereoisomers $\mathbf{b}$ and $\mathbf{d}$ which should be generated by the $\mathbf{T S}_{\mathbf{2}}$ transition state. Exceptionally, the substrates $\mathbf{3}$ and $\mathbf{6}$ gave four diastereomers in a unitary ratio (entries 3 and 6).

With the 2-methylcyclohexanone, having the $\mathrm{CH}_{3}$ group closer to the reaction centre the carbanions attack seems to follow preferably the less steric hindered route $\boldsymbol{\alpha}$ (Scheme 1) of anti type towards the cyclohexanic methyl. The sole diastereoisomers isolated were predominantly of $\mathbf{a}$ and $\mathbf{b}$ structures (Table 1, entries 8-14) afforded by an anti attack of the carbanion to the electrophile (route $\alpha$ ). Among $\mathbf{a}$ and $\mathbf{b}$ structures, the diastereoisomer $\mathbf{a}$, of trans configuration, was mostly obtained, which should be generated by the less hindered $\mathbf{T S}_{\mathbf{1}}$ transition state. Only the substrate 5 produced also a small amount of the diastereoisomer c (entry 12), while the carbanion $\mathbf{6}$ gave, together with $\mathbf{a}$ and $\mathbf{b}$, the structures $\mathbf{c}$ and $\mathbf{d}$ but in much lower yields (entry 13). The heteroarylalkyllithium $\mathbf{1}$, instead, showed a complete diastereoselectivity (entry 8): the sole product 2a of trans configuration was isolated, clearly generated by the $\alpha$ route through the $\mathbf{T S}_{\mathbf{1}}$ transition state. The different isomeric distribution could be justified by a different internal coordination of lithium by the nitrogen and the oxygen atoms, depending on the various heterocyclic moiety among the transition states. 
In order to extend the above results, a few coupling reactions, leading to a sole diastereoisomer or to a diastereoisomeric mixture separable by chromatography, were carried out also with the enantiopure (R)-3-methylcyclohexanone, $(R)$-2-methylcyclohexanone, and (S)-2methylcyclohexanone affording heteroaryloxiranes optically pure. The results and the optical rotation measurements are reported in Table 2.

Table 2. Summary of coupling reactions between $\alpha$-chloroheteroarylalkyllithiums and chiral methylcyclohexanones

\begin{tabular}{|c|c|c|c|c|}
\hline Entry & Substrate & Electrophile & Products & {$[\alpha]_{\mathrm{D}}^{22}$} \\
\hline \multirow{2}{*}{1} & \multirow{2}{*}{1} & \multirow{2}{*}{$(R)$-3-methylcyclohexanone } & $(-)-1 \mathbf{a}$ & -5.8 \\
\hline & & & $(-)-1 c$ & -1.1 \\
\hline \multirow{2}{*}{2} & \multirow{2}{*}{2} & \multirow{2}{*}{ (R)-3-methylcyclohexanone } & $(-)-\mathbf{3 a}$ & -21.9 \\
\hline & & & $(+)-3 c$ & +10.2 \\
\hline 3 & 1 & (R)-2-methylcyclohexanone & $(-)-\mathbf{2 a}$ & -9.8 \\
\hline 4 & 1 & (S)-2-methylcyclohexanone & $(+)-\mathbf{2 a}$ & +10.5 \\
\hline \multirow{2}{*}{5} & \multirow{2}{*}{3} & \multirow{2}{*}{ (S)-2-methylcyclohexanone } & $(+)-6 \mathbf{a}$ & +10.3 \\
\hline & & & $(+)-6 b$ & +5.3 \\
\hline
\end{tabular}

The substrate $\mathbf{1}$ in the coupling reaction with optically pure (S)-2-methylcyclohexanone (Table 2, entry 4) gave the oxirane enantiomerically pure $(+)-\mathbf{2 a}$, containing three stereogenic centres, which absolute configuration was assigned by X-ray measurements (Figure 1). ${ }^{11}$ The asymmetric carbons of the epoxidic ring have $2 S$ and $3 R$ absolute configuration, respectively. Analogously, $\mathbf{1}$ in the coupling reaction with optically pure $(R)$-2-methylcyclohexanone (entry 3 ), produced the other enantiomer (-)-2a, having the epoxidic ring carbons of $2 R$ and $3 S$ absolute configuration, respectively, showing the same ${ }^{1} \mathrm{H}$ and ${ }^{13} \mathrm{C} \mathrm{NMR}$ data of (+)-2a, and according to polarimetric and HPLC measurements. The heteroarylalkyllithium 3 afforded, instead, with $(S)$ 2-methylcyclohexanone (entry 5), two diastereoisomers optically pure, separable by chromatography: (+)-6a, with the absolute configuration equal to (+)-2a, and (+)-6b having both the oxiranic carbons of $R$ absolute configuration. Moreover, the substrates $\mathbf{1}$ and $\mathbf{2}$ reacted with (R)-3-methylcyclohexanone to afford the diastereoisomers a and $\mathbf{c}$ (entries 1, and 2), separable by chromatography, and enantiomerically pure in a ratio of $1: 1$, similarly to what previously reported in Table 1, entries 1 and 2. 


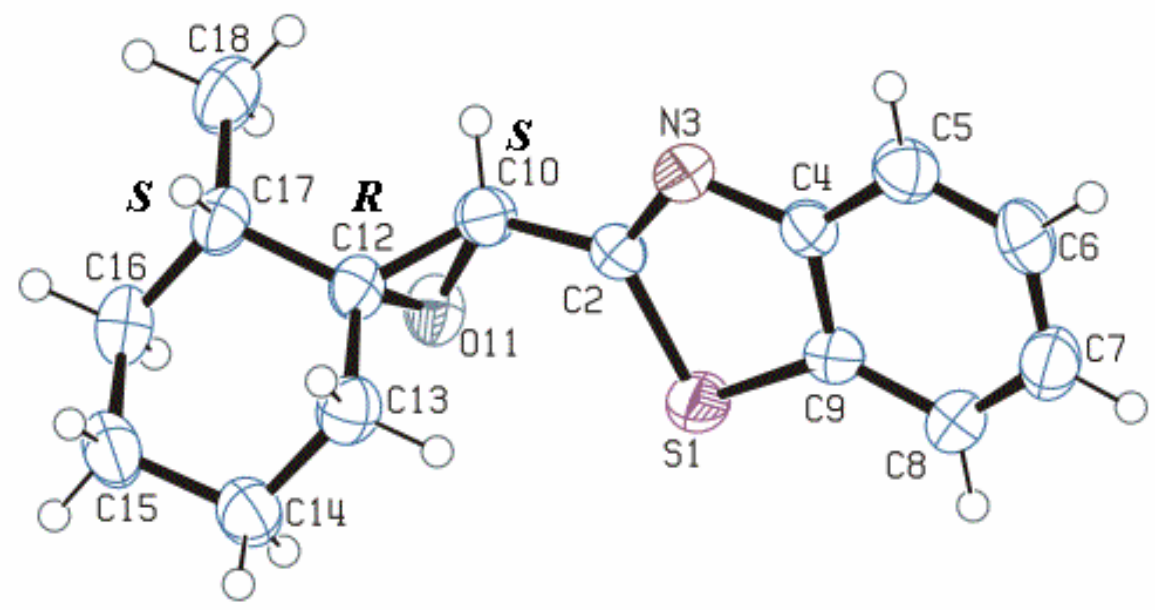

Figure 1. ORTEP III view of compound (+)-2a.

The reaction of 1 with the racemic 2-methylcyclohexanone (Table 1, entry 8) was performed also in the presence of an external chiral ligand, (-)-sparteine, which favours the generation of the enantiomer showing a positive optical rotation, with an enantiomeric ratio of 65:35.

The trans and cis relative configurations for all the above compounds were assigned in comparison with the structure (+)-2a, which was assigned by X-ray measurements, and considering also the oxiranic oxygen atom and the heterocycle influence on the chemical shift of the cyclohexanic $\mathrm{CH}_{3}$. An heteroatom having a lone pair deshields the protons lying in the Van der Waals radius, therefore the $\delta_{\mathrm{CH} 3}$ of the structures $\mathbf{a}$ and $\mathbf{b}$ is always bigger than that showed by the structures $\mathbf{c}$ and $\mathbf{d}$, having, these latter, the farthest oxygen atom. Moreover, the differences between the structures $\mathbf{a}$ and $\mathbf{b}$, and $\mathbf{c}$ and $\mathbf{d}$ were ascribed to the anisotropic effect exerted by the different positions of the heterocycles.

\section{Conclusions}

a-Chloroheteroarylalkyllithiums 1-7, easily available by lithiation of chloroheteroarylalkanes, react as Darzens-type reagents with racemic and enantiomerically pure methylcyclohexanones to form substituted heteroaryloxiranes in a stereoselective way. The structures of the various diastereoisomers and enantiomers isolated were supported by a reliable mechanism. The option of freeing the masked acyl group of the heterocyclic moieties, together with the presence of an oxiranic ring give a remarkable synthetic interest to the isolated compounds, which are useful intermediates for more complicated organic syntheses. 


\section{Experimental Section}

General Procedures. $n$-BuLi was bought as a commercial solution in hexanes (Aldrich) and titrated with N-pivaloyl-o-toluidine prior to use. ${ }^{12}$ THF, toluene, $(-)$-sparteine, $( \pm)-3-$ methylcyclohexanone, $( \pm)$-2-methylcyclohexanone, (R)-(+)-3-methylcyclohexanone, lithium diisopropylamide and other starting materials were of commercial grade (Aldrich) and they were used without further purification. (R)-(-)-2-Methylcyclohexanone and (S)-(+)-2methylcyclohexanone were prepared by enzymatic reduction of $( \pm)-2$-methylcyclohexanone ${ }^{13}$ and subsequent oxidation of enantiomerically pure alcohols with Jones's reagent, following known synthetic protocols. ${ }^{14}$ Petroleum ether refers to the $40-60{ }^{\circ} \mathrm{C}$ boiling fraction. The ${ }^{1} \mathrm{H}$ and the ${ }^{13} \mathrm{C}$ NMR spectra were recorded on a Bruker Advance 400 apparatus $(400.13 \mathrm{MHz}$ and 100.62 MHz, for ${ }^{1} \mathrm{H}$ and ${ }^{13} \mathrm{C}$, respectively) and a Bruker $\mathrm{AC} 200$ apparatus (200 $\mathrm{MHz}$ and 50.3 $\mathrm{MHz}$, for ${ }^{1} \mathrm{H}$ and ${ }^{13} \mathrm{C}$, respectively); with $\mathrm{CDCl}_{3}$ as solvent and TMS as internal standard $(\delta=$ 7.24 for ${ }^{1} \mathrm{H}$ spectra; $\delta=77.0$ for ${ }^{13} \mathrm{C}$ spectra). The IR spectra were recorded on a Perkin Elmer spectrometer Model 283. GC-MS analyses were performed with a Shimadzu GC-17A gas chromatograph (5\% diphenyl / 95\% dimethylpolysiloxane capillary column, $30 \mathrm{~m}, 0.25 \mathrm{~mm}$ i.d.), equipped with a Shimadzu GCMS-QP5050A mass-selective detector operating at $70 \mathrm{eV}$ (EI). HPLC analyses were performed with a Perkin-Elmer series 10 Liquid Chromatograph equipped with an UV-Vis $(254 \mathrm{~nm}$ ) detector and a chiral column (Chiral cell OB-H, $25 \mathrm{~cm}, 0.46 \mathrm{~cm}$ i.d.). Eluent mixtures used for HPLC were $n$-hexane and $n$-hexane/ethanol. Polarimeter Jasco P-1020 was used for polarimetric measurements. Melting points were determined using an electrothermal melting point apparatus and are uncorrected. TLC were performed on Merck silica gel plates with F-254 indicator; viewing was by UV light $(254 \mathrm{~nm})$. Column chromatographies were performed on silica gel (63-200 $\mu \mathrm{m})$ using petroleum ether/diethyl ether $\left(\mathrm{Et}_{2} \mathrm{O}\right)$ mixture as eluents. All reactions involving air-sensitive reagents were performed under nitrogen in oven-dried glassware using syringe/septum cap techniques.

\section{General procedure for the preparation of heteroarylalkyloxiranes}

Method A. used for entries 1-5, and 8-12 of Table 1. A stirred solution of $2 \mathrm{mmol}$ of 2-(1chloroalkyl)benzothiazole, ${ }^{15-18}$ or 2-(1-chloroalkyl)-4-methylthiazole, ${ }^{2}$ in THF $(30 \mathrm{~mL})$, at $78^{\circ} \mathrm{C}$ was treated with $n$-BuLi in hexane $(2.5 \mathrm{M}, 1 \mathrm{~mL}, 2.5 \mathrm{mmol})$, under $\mathrm{N}_{2}$. 2- or 3methylcyclohexanone $(2 \mathrm{mmol})$ in $5 \mathrm{~mL}$ of THF was then added dropwise. The reaction mixtures were kept at $-78^{\circ} \mathrm{C}$ for $30 \mathrm{~min}$, and then warmed up and kept to room temperature for $4 \mathrm{~h}$. The mixtures were then quenched with $10 \mathrm{~mL}$ of a saturated aqueous $\mathrm{NH}_{4} \mathrm{Cl}$ solution, and extracted with $\mathrm{Et}_{2} \mathrm{O}(3 \times 20 \mathrm{~mL})$. The combined organic layers were dried over $\mathrm{Na}_{2} \mathrm{SO}_{4}$ and concentrated in vacuum. The crude products were purified by column chromatography (silica gel, petroleum ether/ $/ \mathrm{Et}_{2} \mathrm{O}, 9: 1$, excepte 14a and $\mathbf{1 4 b}$ eluted with petroleum ether/ $/ \mathrm{Et}_{2} \mathrm{O}, 7: 3$ ) to afford pure heteroarylalkyloxiranes, yields: 50-95\%.

Method B. used for entries 6, 7, 13 and 14 . To $10 \mathrm{~mL}$ of THF, a solution of $1.25 \mathrm{~mL}$ of LDA in hexanes $(2.0 \mathrm{M}, 2.5 \mathrm{mmol})$ was added, at $0{ }^{\circ} \mathrm{C}$ under $\mathrm{N}_{2}$. The mixture was cooled to $-78{ }^{\circ} \mathrm{C}$ and, 
after $30 \mathrm{~min}$., a solution of $2 \mathrm{mmol}$ of 2-(1-chloroalkyl)-4,4-dimethyl-4,5-dihydro-oxazole ${ }^{16-18}$ and $2 \mathrm{mmol}$ of 2 - or 3-methylcyclohexanone in THF $(10 \mathrm{~mL})$ was added dropwise. The reaction mixtures were kept at $-78{ }^{\circ} \mathrm{C}$ for $30 \mathrm{~min}$, and then warmed up and kept at room temperature for 4h. The mixtures were then quenched with $10 \mathrm{~mL}$ of a saturated aqueous $\mathrm{NH}_{4} \mathrm{Cl}$ solution, and extracted with $\mathrm{Et}_{2} \mathrm{O}(3 \times 20 \mathrm{~mL})$. The combined organic layers were dried over $\mathrm{Na}_{2} \mathrm{SO}_{4}$ and concentrated in vacuum. The crude products were purified by column chromatography (silica gel, petroleum ether/ $\mathrm{Et}_{2} \mathrm{O}, 7: 3$ ) to afford the pure oxazolinyloxiranes (oils), yields: 65-80\%.

2-(5-Methyl-1-oxa-spiro[2.5] oct-2-yl)benzothiazole (1a-d). Overall yield $492 \mathrm{mg}$, (95\%). 1a: $226 \mathrm{mg}$ (44\%), white solid, m.p. 88-89 ${ }^{\circ} \mathrm{C}$ (n-hexane); ${ }^{1} \mathrm{H}$ NMR $(200 \mathrm{MHz}) \delta 0.98(\mathrm{~d}, J=6.4$ $\mathrm{Hz}, 3 \mathrm{H}), 1.25-1.92(\mathrm{~m}, 9 \mathrm{H}), 4.22$ (s, $1 \mathrm{H}), 7.34-7.53$ (m, $2 \mathrm{H}), 7.87$ (d, J = 7.9 Hz, $1 \mathrm{H}), 8.00$ (d, $J=7.5 \mathrm{~Hz}, 1 \mathrm{H}) ;{ }^{13} \mathrm{C} \mathrm{NMR}(50.3 \mathrm{MHz}) \delta 22.0,23.3,27.8,30.7,33.8,42.6,62.3,67.2,121.35$, 122.8, 125.0, 126.0, 134.4, 153.5, 168.1; GC-MS (70 eV) m/z (\%) $259\left(\mathrm{M}^{+}, 85\right), 242$ (77), 230 (40), 202 (60), 164 (100), 136 (45); IR $\left(\mathrm{CHCl}_{3}\right) \vee(\sim)=3040,3010,2950,2870,1520,1430$, $1210 \mathrm{~cm}^{-1}$. Anal. Calcd. for $\mathrm{C}_{15} \mathrm{H}_{17} \mathrm{NOS}$ : C, 69.46; H, 6.61; N, 5.40. Found: C, 69.32; H, 6.65; N, 5.34. (-)-1a: $[\alpha]_{\mathrm{D}}{ }^{22}=-5.8\left(0.01, \mathrm{CHCl}_{3}\right)$. 1c: $226 \mathrm{mg}(44 \%)$, white solid, m.p. $87-89{ }^{\circ} \mathrm{C}(n-$ hexane); ${ }^{1} \mathrm{H}$ NMR $(200 \mathrm{MHz}) \delta 0.86(\mathrm{~d}, J=6.4 \mathrm{~Hz}, 3 \mathrm{H}), 1.20-1.96(\mathrm{~m}, 9 \mathrm{H}), 4.22(\mathrm{~s}, 1 \mathrm{H}), 7.35-$ $7.52(\mathrm{~m}, 2 \mathrm{H}), 7.90(\mathrm{~d}, J=7.9 \mathrm{~Hz}, 1 \mathrm{H}), 8.00(\mathrm{~d}, J=8.0 \mathrm{~Hz}, 1 \mathrm{H}) ;{ }^{13} \mathrm{C} \mathrm{NMR}(50.3 \mathrm{MHz}) \delta 22.0$, 23.4, 30.6, 33.9, 34.0, 36.5, 62.2, 67.5, 121.5, 122.8, 125.0, 126.0, 134.4, 153.5, 168.1; GC-MS $(70 \mathrm{eV}) \mathrm{m} / \mathrm{z}(\%) 259\left(\mathrm{M}^{+}, 85\right), 242$ (77), 230 (35), 202 (58), 164 (100), 136 (30); IR $\left(\mathrm{CHCl}_{3}\right)$ $v(\sim)$ 3040, 3010, 2950, 2870, 1520, 1430, $1210 \mathrm{~cm}^{-1}$. Anal. Calcd. for $\mathrm{C}_{15} \mathrm{H}_{17} \mathrm{NOS}$ : C, 69.46; H, 6.61; N, 5.40. Found: C, 69.75; H, 6.60; N, 5.48. (-)-1c: $[\alpha]_{\mathrm{D}}{ }^{22}=-1.1\left(0.04, \mathrm{CHCl}_{3}\right) . \mathbf{1 b}$ and 1d were isolated in traces and identified by GC-MS. 1b: GC-MS $(70 \mathrm{eV}) \mathrm{m} / \mathrm{z}(\%) 259\left(\mathrm{M}^{+}, 88\right)$, 242 (77), 230 (42), 202 (56), 164 (100), 136 (39). 1d: GC-MS (70 eV) m/z (\%) 259 (M+ 80), 242 (77), 230 (40), 202 (50), 164 (100), 136 (35).

2-(4-Methyl-1-oxa-spiro[2.5]oct-2-yl)benzothiazole (2a). Overall yield $492 \mathrm{mg}$ (95\%), white solid, m.p. 105-106 ${ }^{\circ} \mathrm{C}$ (n-hexane); ${ }^{1} \mathrm{H}$ NMR (200 MHz) $\delta 1.00(\mathrm{~d}, J=6.7 \mathrm{~Hz}, 3 \mathrm{H}), 1.45-1.95$ $(\mathrm{m}, 9 \mathrm{H}), 4.35(\mathrm{~s}, 1 \mathrm{H}), 7.34-7.53(\mathrm{~m}, 2 \mathrm{H}), 7.88(\mathrm{~d}, J=7.8 \mathrm{~Hz}, 1 \mathrm{H}) 8.00(\mathrm{~d}, J=8.0 \mathrm{~Hz}, 1 \mathrm{H})$; ${ }^{13} \mathrm{C}$ NMR $(50.3 \mathrm{MHz}) \delta 14.3,23.2,24.5,27.3,32.6,36.1,60.8,69.7,121.6,122.9,125.0,126.1$, 134.7, 153.6, 168.8; GC-MS (70 eV) m/z (\%) $259\left(\mathrm{M}^{+}, 65\right), 244$ (40), 228 (51), 202 (32), 164 (100), 149 (70), 136 (50) IR $\left(\mathrm{CHCl}_{3}\right) v(\sim)$ 3060, 2920, 2860, 1510, 1440, $1200 \mathrm{~cm}^{-1}$. Anal. Calcd. for $\mathrm{C}_{15} \mathrm{H}_{17} \mathrm{NOS}$ : C, 69.46; H, 6.61; N, 5.40. Found: C, 70.18; H, 6.62; N, 5.30. (-)-2a: $[\alpha]_{\mathrm{D}}^{22}=-9.8\left(0.01, \mathrm{CHCl}_{3}\right) .(+)-2 \mathrm{a}:[\alpha]_{\mathrm{D}}^{22}=+10.5\left(0.02, \mathrm{CHCl}_{3}\right)$.

2-(2,5-Dimethyl-1-oxa-spiro[2.5] oct-2-yl)benzothiazole (3a, 3c). Overall yield $355 \mathrm{mg}$ (65\%). 3a: $177 \mathrm{mg}$ (32\%), yellow oil; ${ }^{1} \mathrm{H}$ NMR (400.13 MHz) $\delta \quad 0.99(\mathrm{~d}, J=6.6 \mathrm{~Hz}, 3 \mathrm{H}), 1.25-1.85$ $(\mathrm{m}, 12 \mathrm{H}), 7.36-7.48(\mathrm{~m}, 2 \mathrm{H}), 7.85-7.88(\mathrm{~m}, 1 \mathrm{H}), 7.99-8.01(\mathrm{~m}, 1 \mathrm{H}) ;{ }^{13} \mathrm{C} \mathrm{NMR}(100.62 \mathrm{MHz})$ $\delta 18.5,22.4,23.2,29.8,30.7,33.9,38.5,66.1,69.9,121.5,122.8,124.9,125.9,134.6,153.6$, 173.6; GC-MS (70 eV) m/z (\%) $273\left(\mathrm{M}^{+}, 55\right), 258$ (15), 256 (30), 230 (22), 178 (100), 136 (42); IR (film) $v(\sim) 3060,2920,2875,1520,1440,760,730,700 \mathrm{~cm}^{-1} .(-)-3 a:[\alpha]_{\mathrm{D}}^{22}=-21.9(0.01$, $\mathrm{CHCl}_{3}$ ). 3c: $177 \mathrm{mg}(32 \%)$, yellow oil; ${ }^{1} \mathrm{H}$ NMR (400.13 MHz) $\delta \quad 0.79(\mathrm{~d}, J=6.4 \mathrm{~Hz}, 3 \mathrm{H})$, 
1.20-1.85 (m, $12 \mathrm{H}), 7.36-7.50(\mathrm{~m}, 2 \mathrm{H}), 7.85-7.89$ (m, $1 \mathrm{H}), 7.96-8.01(\mathrm{~m}, 1 \mathrm{H}) ;{ }^{13} \mathrm{C}$ NMR $(100.62 \mathrm{MHz}) \delta 18.6,22.2,23.6,29.6,30.5,34.1,38.5,66.1,70.9,121.7,122.9,125.9,126.0$, 134.7, 153.6, 173.6; GC-MS (70 eV) m/z (\%) $273\left(\mathrm{M}^{+}, 94\right), 258$ (55), 256 (80), 230 (75), 178 (100), 139 (90); IR (film) $v(\sim) 3060,2920,2875,1520,1440,760,730,700 \mathrm{~cm}^{-1} .(+)-3 \mathrm{c}:[\alpha]_{\mathrm{D}}{ }^{22}$ $=+10.2\left(0.01, \mathrm{CHCl}_{3}\right)$.

2-(2,4-Dimethyl-1-oxa-spiro[2.5]oct-2-yl)benzothiazole (4a, 4b). Overall yield $491 \mathrm{mg}(90 \%)$; 4a: $393 \mathrm{mg}$ (72\%), white solid, m.p. 83-85 ${ }^{\circ} \mathrm{C}$ (n-hexane); ${ }^{1} \mathrm{H}$ NMR $(400.13 \mathrm{MHz}) \delta 1.10(\mathrm{~d}, J$ $=7.1 \mathrm{~Hz}, 3 \mathrm{H}), 1.42-1.93(\mathrm{~m}, 9 \mathrm{H}), 1.87(\mathrm{~s}, 3 \mathrm{H}), 7.38-7.52(\mathrm{~m}, 2 \mathrm{H}), 7.89(\mathrm{~d}, J=8.1 \mathrm{~Hz}, 1 \mathrm{H})$, $8.05(\mathrm{~d}, J=8.0 \mathrm{~Hz}, 1 \mathrm{H}) ;{ }^{13} \mathrm{C}$ NMR $(50.3 \mathrm{MHz}) \delta 14.1,18.9,19.4,25.7,25.8,30.7,32.0,67.0$, 73.0, 121.6, 122.9, 124.9, 126.0, 134.5, 153.9, 169.0; GC-MS (70 eV) $\mathrm{m} / \mathrm{z}(\%) 273\left(\mathrm{M}^{+}, 14\right)$, 216 (14), 178 (100), 162 (17), 136 (25); IR $\left(\mathrm{CHCl}_{3}\right) v(\sim)$ 3060, 2920, 2850, 1450, 1370, 1320, 1240, 1120, 1040, 950, 760, $730 \mathrm{~cm}^{-1}$. Anal. Calcd. for $\mathrm{C}_{16} \mathrm{H}_{19} \mathrm{NOS}$ : C, 70.29; H, 7.00; N, 5.12 . Found: C, 70.35; H, 6.97; N, 5.21. 4b: $98 \mathrm{mg}(18 \%)$, yellow oil; ${ }^{1} \mathrm{H}$ NMR $(400.13 \mathrm{MHz}) \delta 1.21$ (d, $J=7.1 \mathrm{~Hz}, 3 \mathrm{H}), 1.42-1.93$ (m, $9 \mathrm{H}), 1.88$ (s, 3H), 7.38-7.52 (m, $2 \mathrm{H}), 7.89$ (d, $J=8.1 \mathrm{~Hz}, 1$ $\mathrm{H}), 8.05(\mathrm{~d}, J=8.0 \mathrm{~Hz}, 1 \mathrm{H}) ;{ }^{13} \mathrm{C}$ NMR $(50.3 \mathrm{MHz}) \delta 15.3,18.8,19.0,25.8,30.7,32.1,32.2$, 67.0, 73.0, 121.6, 122.9, 124.9, 126.0, 134.5, 153.9, 169.0; GC-MS (70 eV) m/z (\%) $273\left(\mathrm{M}^{+}\right.$, 34), 216 (20), 178 (100), 162 (17), 136 (30); IR (film) v( ) 2930, 2860, 1450, 1370, 1240, 1120, $1050,950,760,730 \mathrm{~cm}^{-1}$.

4-Methyl-2-(5-methyl-1-oxa-spiro[2.5]oct-2-yl)thiazole (5a-d). Overall yield $223 \mathrm{mg}$ (50\%). $\mathbf{5 a}+\mathbf{5 b}$ : $111 \mathrm{mg}$ (25\%), yellow oil, inseparable mixture of two diastereoisomers $(\mathrm{dr}=25: 25) ;{ }^{1} \mathrm{H}$ NMR $(400.13 \mathrm{MHz}) \delta 0.95(\mathrm{~d}, J=6.6 \mathrm{~Hz}, 3 \mathrm{H}), 0.98(\mathrm{~d}, J=6.5 \mathrm{~Hz}, 3 \mathrm{H}), 1.30-1.90(\mathrm{~m}, 18 \mathrm{H})$, $2.44(\mathrm{~s}, 3 \mathrm{H}), 2.45(\mathrm{~s}, 3 \mathrm{H}), 4.06(\mathrm{~s}, 1 \mathrm{H}), 4.09(\mathrm{~s}, 1 \mathrm{H}), 6.83(\mathrm{~s}, 1 \mathrm{H}), 6.84(\mathrm{~s}, 1 \mathrm{H}) ;{ }^{13} \mathrm{C}$ NMR $(100.62$ MHz) $\delta 17.4,17.5,22.4,22.6,23.8,24.3,28.1,28.6,30.1,31.1,32.8,34.1,34.4,43.1,43.9$, 62.8, 67.4, 67.7, 113.7, 113.8, 153.6, 153.7, 166.7, 166.9; GC-MS (70 eV) m/z (\%) $223\left(\mathrm{M}^{+}\right.$, 12), 207 (60), 192 (100), 164 (40), 128 (30); IR (film) v( ) 3100, 2910, 2840, 1520, 1200, 800, $730 \mathrm{~cm}^{-1} . \mathbf{5 c}+5 \mathrm{~d}$ : $111 \mathrm{mg}(25 \%)$, yellow oil, inseparable mixture of two diastereoisomers $(\mathrm{dr}=$ 25:25); ${ }^{1} \mathrm{H}$ NMR $(200 \mathrm{MHz}) \delta 0.83(\mathrm{~d}, J=5.9 \mathrm{~Hz}, 3 \mathrm{H}), 0.87(\mathrm{~d}, J=6.3 \mathrm{~Hz}, 3 \mathrm{H}), 1.30-1.85(\mathrm{~m}$, $18 \mathrm{H}), 2.43$ (s, 3H), $2.46(\mathrm{~s}, 3 \mathrm{H}), 4.06(\mathrm{~s}, 1 \mathrm{H}), 4.10(\mathrm{~s}, 1 \mathrm{H}), 6.85(\mathrm{~s}, 1 \mathrm{H}), 6.87(\mathrm{~s}, 1 \mathrm{H}) ;{ }^{13} \mathrm{C} \mathrm{NMR}$ (100.62 MHz) $\delta 17.4,17.5,22.5,22.6,23.8,24.8,29.4,30.1,31.1,31.9,34.1,34.4,34.5,35.2$, 36.7, 62.8, 67.3, 67.7, 113.7, 113.8, 153.2, 153.6, 166.9, 167.0; GC-MS (70 eV) m/z (\%) 223 $\left(\mathrm{M}^{+}, 16\right), 207$ (65), 192 (100), 164 (70), 128 (50); IR (film) $v(\sim)$ 3100, 2910, 2840, 1520, 1200, $800,730 \mathrm{~cm}^{-1}$.

4-Methyl-2-(4-methyl-1-oxa-spiro[2.5]oct-2-yl)thiazole (6a, 6b). Overall yield $424 \mathrm{mg}$ (95\%). 6a: $285 \mathrm{mg}$ (64\%), white solid, m.p. $60-61{ }^{\circ} \mathrm{C}$ ( $n$-hexane); ${ }^{1} \mathrm{H}$ NMR $(200 \mathrm{MHz}) \delta 0.97$ (d, $J=$ $6.8 \mathrm{~Hz}, 3 \mathrm{H}), 1.40-190(\mathrm{~m}, 9 \mathrm{H}), 2.44$ (d, $J=0.8 \mathrm{~Hz}, 3 \mathrm{H}), 4.22$ (s, $1 \mathrm{H}), 6.80$ (q, $J=0.8 \mathrm{~Hz}, 1$ $\mathrm{H}) ;{ }^{13} \mathrm{C}$ NMR $(50.3 \mathrm{MHz}) \delta 14.2,17.0,23.2,24.5,27.0,32.5,35.9,60.7,69.2,113.2,153.0$, 166.8; GC-MS (70 eV) m/z (\%) $223\left(\mathrm{M}^{+}, 32\right), 208$ (34), 192 (38), 180 (20), 128 (100), 113 (90), 100 (57); IR $\left(\mathrm{CHCl}_{3}\right) v(\sim) 3100,2910,2840,1520,1200,800,730 \mathrm{~cm}^{-1}$. Anal. Calcd. for $\mathrm{C}_{12} \mathrm{H}_{17} \mathrm{NOS}$ : C, 64.54; H, 7.67; N, 6.27. Found: C, 64.85; H, 7.65; N 6.30. (+)-6a: $[\alpha]_{\mathrm{D}}^{22}=+10.3$, (0.03, $\left.\mathrm{CHCl}_{3}\right)$. 6b: $139 \mathrm{mg}(31 \%)$, white solid, m.p. $44-45{ }^{\circ} \mathrm{C}$ ( $n$-hexane); ${ }^{1} \mathrm{H}$ NMR (200 MHz) 
$\delta 1.12(\mathrm{~d}, J=7.2 \mathrm{~Hz}, 3 \mathrm{H}), 1.20-2.18(\mathrm{~m}, 9 \mathrm{H}), 2.45(\mathrm{~d}, J=0.7 \mathrm{~Hz}, 3 \mathrm{H}), 4.07(\mathrm{~s}, 1 \mathrm{H}), 6.80(\mathrm{q}$, $J=0.8 \mathrm{~Hz}, 1 \mathrm{H}) ;{ }^{13} \mathrm{C}$ NMR $(50.3 \mathrm{MHz}) \delta 14.3,17.1,19.1,25.7,29.8,30.3,30.8,63.7,69.9$, 113.3, 153.1, 166.8; GC-MS (70 eV) m/z (\%) 223 (M+27), 206 (33), 192 (41), 128 (100), 113 (96), 100 (53); IR $\left(\mathrm{CHCl}_{3}\right) v(\sim) 3100,2910,2840,1520,1200,800,730 \mathrm{~cm}^{-1}$. Anal. Calcd. for $\mathrm{C}_{12} \mathrm{H}_{17} \mathrm{NOS}$ : C, 64.54; H, 7.67; N, 6.27. Found: C, 64.90; H, 7.60; N, 6.25. (+)-6b: $[\alpha]_{\mathrm{D}}{ }^{22}=+5.3$, $\left(0.04, \mathrm{CHCl}_{3}\right)$.

4-Methyl-2-(2,5-dimethyl-1-oxa-spiro[2.5]oct-2-yl)thiazole (7a-d). Overall yield $427 \mathrm{mg}$ $(90 \%)$, inseparable mixture of four diastereoisomers, yellow oil, distinguishable by NMR and GC-MS, $(\mathrm{dr}=43: 7: 43: 7) .7 \mathbf{a}:(39 \%) ;{ }^{1} \mathrm{H}$ NMR $(200 \mathrm{MHz}) \delta 0.97(\mathrm{~d}, J=6.3 \mathrm{~Hz}, 3 \mathrm{H}), 1.10-1.76$ (m, $9 \mathrm{H}), 1.73$ (s, $3 \mathrm{H}), 2.44$ (d, $J=0.9 \mathrm{~Hz}, 3 \mathrm{H}), 6.78$ (q, $J=0.9 \mathrm{~Hz}, 1 \mathrm{H}) ;{ }^{13} \mathrm{C}$ NMR $(50.3 \mathrm{MHz})$ $\delta$ 17.0, 18.7, 22.4, 23.3, 29.5, 30.7, 34.0, 38.6, 65.9, 69.8, 112.9, 152.8, 171.8; GC-MS (70 eV) $\mathrm{m} / \mathrm{z}(\%) 237\left(\mathrm{M}^{+}, 26\right), 222$ (11), 220 (14), 194 (15), 142 (100), 126 (12), 100 (22). 7b: (6\%); ${ }^{1} \mathrm{H}$ NMR $(200 \mathrm{MHz}) \delta 0.99(\mathrm{~d}, J=6.3 \mathrm{~Hz}, 3 \mathrm{H}), 1.10-1.76(\mathrm{~m}, 9 \mathrm{H}), 1.76(\mathrm{~s}, 3 \mathrm{H}), 2.44$ (d, $J=0.9$ $\mathrm{Hz}, 3 \mathrm{H}), 6.78(\mathrm{q}, J=0.9 \mathrm{~Hz}, 1 \mathrm{H}) ;{ }^{13} \mathrm{C} \mathrm{NMR}(50.3 \mathrm{MHz}) \delta 15.3,17.9,22.0,23.8,30.5,32.1$, 33.2, 39.0, 65.0, 70.0, 112.9, 152.8, 171.8; GC-MS (70 eV) m/z (\%) $237\left(\mathrm{M}^{+}, 30\right), 222$ (15), 220 (10), 194 (15), 142 (100), 126 (15), 100 (20). 7c: (39\%); ${ }^{1} \mathrm{H}$ NMR (200 MHz) $\delta 0.83$ (d, $J=6.4$ $\mathrm{Hz}, 3 \mathrm{H}), 1.18-1.80$ (m, $9 \mathrm{H}), 1.74$ (s, $3 \mathrm{H}), 2.46$ (d, $J=0.9 \mathrm{~Hz}, 3 \mathrm{H}), 6.80$ (q, $J=0.9 \mathrm{~Hz}, 1 \mathrm{H}$ ); ${ }^{13} \mathrm{C}$ NMR (50.3 MHz) $\delta 15.3,17.7,18.8,22.2,23.5,29.6,30.4,34.0,63.7,65.9,113.0,152.5$, 171.5; GC-MS (70 eV) m/z (\%) 237 (M+, 20), 222 (12), 220 (20), 194 (10), 142 (100), 126 (12), 100 (22). 7d: (6\%); ${ }^{1} \mathrm{H}$ NMR $(200 \mathrm{MHz}) \delta 0.86(\mathrm{~d}, J=6.4 \mathrm{~Hz}, 3 \mathrm{H}), 1.18-1.80(\mathrm{~m}, 9 \mathrm{H}), 1.75$ (s, $3 \mathrm{H}), 2.46(\mathrm{~d}, J=0.9 \mathrm{~Hz}, 3 \mathrm{H}), 6.80(\mathrm{q}, J=0.9 \mathrm{~Hz}, 1 \mathrm{H}) ;{ }^{13} \mathrm{C} \operatorname{NMR}(50.3 \mathrm{MHz}) \delta 14.0,17.0$, 18.0, 22.4, 22.3, 29.8, 31.8, 38.2, 63.7, 65.0, 113.0, 152.5, 171.5; GC-MS (70 eV) m/z (\%) 237 $\left(\mathrm{M}^{+}, 20\right), 222$ (11), 220 (22), 194 (10), 142 (100), 126 (13), 100 (18); IR was measured on the inseparable mixture, IR (film) $v(\sim)$ 2930, 2860, 1700, 1520, 1450, 1370, 1300, 1140, $1040 \mathrm{~cm}^{-1}$. 4-Methyl-2-(2,4-dimethyl-1-oxa-spiro[2.5]oct-2-yl)thiazole (8a, 8b). Overall yield $332 \mathrm{mg}$ (70\%). 8a: $251 \mathrm{mg}(53 \%)$, yellow oil; ${ }^{1} \mathrm{H}$ NMR (200 MHz) $\delta 1.05$ (d, $\left.J=6.8 \mathrm{~Hz}, 3 \mathrm{H}\right), 1.76$ (s, $3 \mathrm{H}), 1.37-1.99(\mathrm{~m}, 9 \mathrm{H}), 2.44(\mathrm{~d}, J=0.7 \mathrm{~Hz}, 3 \mathrm{H}), 6.78(\mathrm{q}, J=0.7 \mathrm{~Hz}, 1 \mathrm{H}) ;{ }^{13} \mathrm{C}$ NMR $(50.3$ MHz) $\delta 14.1,17.2,19.1,19.3,25.5,25.7,30.5,31.5,66.8,72.8,113.0,152.9,172.0$; GC-MS $(70 \mathrm{eV}) \mathrm{m} / \mathrm{z}(\%) 237\left(\mathrm{M}^{+}, 12\right), 222(8), 142$ (100), 126 (13), 100 (17); IR (film) $v(\sim)$ 2930, 2860, 1450, 1370, $1050 \mathrm{~cm}^{-1} .8 \mathbf{b}: 81 \mathrm{mg}(17 \%)$, yellow oil; ${ }^{1} \mathrm{H}$ NMR $(200 \mathrm{MHz}) \delta 1.14(\mathrm{~d}, J=6.8 \mathrm{~Hz}$, $3 \mathrm{H}), 1.77$ (s, $3 \mathrm{H}), 1.37-1.99(\mathrm{~m}, 9 \mathrm{H}), 2.44(\mathrm{~d}, J=0.7 \mathrm{~Hz}, 3 \mathrm{H}), 6.78$ (q, $J=0.8 \mathrm{~Hz}, 1 \mathrm{H}) ;{ }^{13} \mathrm{C}$ NMR $(50.3 \mathrm{MHz}) \delta 14.2,17.2,18.9,24.9,25.3,31.1,31.9,66.8,72.8,112.9,152.9,172.0$; GCMS (70 eV) m/z (\%) $237\left(\mathrm{M}^{+}, 12\right), 222$ (8), 142 (100), 126 (13), 100 (17); IR (film) $v(\sim)$ 2930, $2860,1450,1370,1050 \mathrm{~cm}^{-1}$.

4-Methyl-2-(5-methyl-2-phenyl-1-oxa-spiro[2.5]oct-2-yl)thiazole (9a, 9c). Overall yield: $388 \mathrm{mg}(65 \%)$. 9a: $194 \mathrm{mg}(32 \%)$, yellow oil; ${ }^{1} \mathrm{H}$ NMR $(200 \mathrm{MHz}) \delta 0.88(\mathrm{~d}, J=6.3 \mathrm{~Hz}, 3 \mathrm{H})$, 1.20-177 (m, 9 H), 2.43 (d, $J=0.9$ Hz, $3 \mathrm{H}$ ), 6.77 (q, $J=0.9 \mathrm{~Hz}, 1 \mathrm{H}), 7.26-7.40$ (m, $3 \mathrm{H}), 7.60$ $7.70(\mathrm{~m}, 2 \mathrm{H}) ;{ }^{13} \mathrm{C}$ NMR $(50.3 \mathrm{MHz}) \delta 17.2,22.4,23.6,30.1,30.8,34.1,39.1,69.3,71.7,113.3$, 127.2, 127.6, 127.9, 128.0, 138.0, 152.8, 173.1; GC-MS (70 eV) m/z (\%) $299\left(\mathrm{M}^{+}, 57\right), 204$ (100), 186 (27), 147 (31), 126 (22), 105 (35); IR (film) v( ) 2920, 2860, 1520, 1490, 1440, 1230, 
1120, 985, 760, $705 \mathrm{~cm}^{-1}$. 9c: $195 \mathrm{mg}(32 \%)$, yellow oil; ${ }^{1} \mathrm{H}$ NMR $(200 \mathrm{MHz}) \delta \quad 0.82(\mathrm{~d}, J=6.3$ Hz, 3 H), 1.20-177 (m, 9 H), 2.43 (d, J=0.9 Hz, 3 H), 6.77 (q, J=0.9 Hz, 1 H), 7.26-7.40 (m, 3 $\mathrm{H}), 7.60-7.70(\mathrm{~m}, 2 \mathrm{H}) ;{ }^{13} \mathrm{C} \mathrm{NMR}(50.3 \mathrm{MHz}) \delta 17.2,22.4,23.5,30.5,30.8,34.0,38.7,69.3$, 71.7, 113.3, 127.2, 127.6, 127.9, 128.0, 138.0, 152.8, 173.1; GC-MS (70 eV) m/z (\%) $299\left(\mathrm{M}^{+}\right.$, 57), 204 (100), 186 (27), 147 (31), 126 (22), 105 (35); IR (film) v( ) 2920, 2860, 1520, 1490, 1440, 1230, 1120, 985, 760, $705 \mathrm{~cm}^{-1}$.

4-Methyl-2-(4-methyl-2-phenyl-1-oxa-spiro[2.5]oct-2-yl)thiazole (10a-c). Overall yield: $431 \mathrm{mg}$ (72\%). 10a: $215 \mathrm{mg}$ (36\%), yellow oil; ${ }^{1} \mathrm{H}$ NMR (400.13 MHz) $\delta 1.08$ (d, J=7.1 Hz, 3 H), 1.16-1.89 (m, 9 H), 2.45 (s, $3 \mathrm{H}), 6.80$ (s, $1 \mathrm{H}), 7.29-7.38(\mathrm{~m}, 4 \mathrm{H}), 7.66$ (d, J = 7.9 Hz, 1 $\mathrm{H}) ;{ }^{13} \mathrm{C}$ NMR $(100.62 \mathrm{MHz}) \delta 15.3,17.7,19.5,20.1,25.1,30.6,32.7,70.4,74.8,113.7,127.7$, 128.3, 128.4, 137.8, 153.6, 169.9; GC-MS (70 eV) m/z (\%) 299 (M+ 50$), 283$ (24), 204 (100), 189 (37), 147 (29); IR (film) v( ) 2920, 2860, 1520, 1490, 1440, 1230, 1120, 985, 760, $705 \mathrm{~cm}^{-1}$. 10b: $150 \mathrm{mg}$ (25\%), white solid, m.p. $126-128{ }^{\circ} \mathrm{C} ;{ }^{1} \mathrm{H} \mathrm{NMR}(400.13 \mathrm{MHz}) \delta 1.14(\mathrm{~d}, J=6.5 \mathrm{~Hz}$, $3 \mathrm{H}), 1.16-1.89$ (m, $9 \mathrm{H}), 2.43$ (s, $3 \mathrm{H}), 6.76$ (s, $1 \mathrm{H}), 7.30-7.38$ (m, $4 \mathrm{H}), 7.80$ (d, J = 7.5 Hz, 1 $\mathrm{H}) ;{ }^{13} \mathrm{C}$ NMR $(100.62 \mathrm{MHz}) \delta 14.8,17.8,24.6,25.7,27.1,31.0,32.2,71.2,75.1,113.6,127.6$, 128.0, 128.7, 137.6, 153.6, 169.5; GC-MS (70 eV) m/z (\%: 299 (M+, 48), 283 (27), 204 (100), 189 (40), 147 (30); IR $\left(\mathrm{CHCl}_{3}\right) v(\sim)$ 2920, 2860, 1520, 1490, 1440, 1230, 1120, 985, 760, $705 \mathrm{~cm}^{-1}$. 10c: $66 \mathrm{mg}(25 \%)$, yellow oil; ${ }^{1} \mathrm{H}$ NMR $(400.13 \mathrm{MHz}) \delta 0.85(\mathrm{~d}, J=7.2 \mathrm{~Hz}, 3 \mathrm{H})$, 1.20-1.90 (m, $9 \mathrm{H}), 2.41$ (s, $3 \mathrm{H}), 6.73(\mathrm{~s}, 1 \mathrm{H}), 7.24-7.33$ (m, $4 \mathrm{H}), 7.74(\mathrm{~d}, J=7.4 \mathrm{~Hz}, 1 \mathrm{H})$; ${ }^{13} \mathrm{C}$ NMR $(100.62 \mathrm{MHz}) \delta 14.9,17.7,20.0,24.6,26.3,30.7,32.6,70.3,75.3,113.7,127.9$, 128.0, 128.1, 136.9, 153.4, 170.0; GC-MS (70 eV) m/z (\%) $299\left(\mathrm{M}^{+}, 55\right), 283$ (23), 204 (100), 189 (40), 147 (29); IR (film) v( ) 2920, 2860, 1520, 1490, 1440, 1230, 1120, 985, 760, $705 \mathrm{~cm}^{-1}$. 4,4-Dimethyl-2-(5-methyl-1-oxa-spiro[2.5]oct-2-yl)-4,5-dihydro-oxazole (11a-d). Overall yield: $357 \mathrm{mg}(80 \%)$. Inseparable mixture of four diastereoisomers, yellow oil, distinguishable only by GC-MS, (dr = 25:25:25:25). 11a: (20\%); GC-MS (70 eV) m/z (\%) $223\left(\mathrm{M}^{+}, 5\right), 208$ (10), 206 (10), 194 (10), 168 (30), 138 (25), 95 (100). 11b: (20\%); GC-MS (70 eV) m/z (\%) 223 $\left(\mathrm{M}^{+}, 10\right), 208$ (8), 206 (15), 194 (12), 168 (35), 138 (25), 95 (100); 11c: (20\%); GC-MS (70 eV) m/z (\%) 223 (M $\left.\mathrm{M}^{+}, 20\right), 208$ (25), 206 (8), 194 (10), 168 (30), 138 (30), 95 (100). 11d: (20\%); GC-MS (70 eV) m/z (\%) $223\left(\mathrm{M}^{+}, 4\right), 208$ (13), 206 (20), 194 (30), 168 (22), 138 (25), 95 (100); IR measured on the inseparable mixture, IR $\left(\mathrm{CHCl}_{3}\right) v(\sim) 2910,2850,1655,1450,1365,1300$, $1090 \mathrm{~cm}^{-1}$.

4,4-Dimethyl-2-(4-methyl-1-oxa-spiro[2.5]oct-2-yl)-4,5-dihydro-oxazole (12a-d). Overall yield $357 \mathrm{mg}(80 \%)$. 12a+12c: $179 \mathrm{mg}(40 \%)$, yellow oil, inseparable mixture of two diastereoisomers distinguishable by ${ }^{1} \mathrm{H}$ NMR and GC-MS $(\mathrm{dr}=38: 12) ; 12 a:(30 \%)$; ${ }^{1} \mathrm{H}$ NMR $(200 \mathrm{MHz}) \delta \quad 0.87(\mathrm{~d}, J=6.8 \mathrm{~Hz}, 3 \mathrm{H}), 1.27(\mathrm{~s}, 6 \mathrm{H}), 1.28-1.80(\mathrm{~m}, 9 \mathrm{H}), 3.51(\mathrm{~s}, 1 \mathrm{H}), 3.96$ (s, $2 \mathrm{H})$; GC-MS (70 eV) m/z (\%) 223 (M+, 3), 206 (5), 192 (12), 168 (27), 138 (15), 113 (15), 95 (100). 12c: (10\%); ${ }^{1} \mathrm{H}$ NMR $(200 \mathrm{MHz}) \delta 0.85(\mathrm{~d}, J=6.7 \mathrm{~Hz}, 3 \mathrm{H}), 1.27(\mathrm{~s}, 6 \mathrm{H}), 1.28-1.81$ (m, $9 \mathrm{H}), 3.46$ (s, $1 \mathrm{H}), 3.96$ (s, $2 \mathrm{H})$; GC-MS (70 eV) m/z (\%) $223\left(\mathrm{M}^{+}, 4\right), 206$ (7), 192 (20), 168 (23), 138 (15), 113 (18), 95 (100). ${ }^{13} \mathrm{C}$ NMR and IR were measured on the mixture; ${ }^{13} \mathrm{C}$ NMR $(50.3 \mathrm{MHz}) \delta 14.2,14.6,23.5,23.9,24.6,25.2,27.3,28.2,28.3,28.4,28.5,32.5,33.3$, 35.6, 
36.6, 52.7, 54.9, 66.8, 67.4, 67.5, 79.2, 79.3, 161.5, 161.6; IR (film) $v(\sim)$ 2920, 2860, 1660, 1520, 1450, $1380 \mathrm{~cm}^{-1} . \mathbf{1 2 b}+\mathbf{1 2 d}$ : $179 \mathrm{mg}(40 \%)$, yellow oil, inseparable mixture of two diastereoisomers distinguishable only by ${ }^{1} \mathrm{H}$ NMR and GC-MS $(\mathrm{dr}=38: 12) ; \mathbf{1 2 b}$ : $(30 \%) ;{ }^{1} \mathrm{H}$ NMR (200 MHz) $\delta 1.12(\mathrm{~d}, J=7.2 \mathrm{~Hz}, 3 \mathrm{H}), 1.29$ (s, $6 \mathrm{H}), 1.30-2.15(\mathrm{~m}, 9 \mathrm{H}), 3.37(\mathrm{~s}, 1 \mathrm{H})$, 4.00 (s, $2 \mathrm{H})$; GC-MS (70 eV) m/z (\%) $223\left(\mathrm{M}^{+}, 5\right), 206$ (10), 192 (8), 168 (25), 138 (20), 113 (15), 95 (100). 12d: (10\%); ${ }^{1} \mathrm{H}$ NMR (200 MHz) $\delta 1.10$ (d, J=7.2 Hz, $\left.3 \mathrm{H}\right), 1.30$ (s, $\left.6 \mathrm{H}\right), 1.30$ 2.15 (m, 9 H), 3.41 (s, $1 \mathrm{H}), 4.00$ (s, $2 \mathrm{H})$; GC-MS (70 eV) m/z (\%) $223\left(\mathrm{M}^{+}, 10\right), 206$ (5), 192 (10), $168(24), 138$ (15), 113 (15), 95 (100). ${ }^{13} \mathrm{C}$ NMR and IR were measured on the mixture; ${ }^{13} \mathrm{C}$ NMR (50.3 MHz): $\delta=14.1,14.3,23.4,23.7,24.2,24.5,25.1,25.8,28.3,28.4,29.7,30.1,30.5$, $30.6,31.1,32.5,69.3,69.9,70.4,70.5,79.4,79.5,161.4,161.5$. IR (film) $v(\sim) 2920,2860,1660$, $1520,1450,1380 \mathrm{~cm}^{-1}$.

4,4-Dimethyl-2-(2,5-dimethyl-1-oxa-spiro[2.5]oct-2-yl)-4,5-dihydro-oxazole (13a-d). Overall yield $308 \mathrm{mg}(65 \%)$. 13a+13c: $174 \mathrm{mg}(58 \%)$, inseparable mixture of two diastereoisomers, yellow oil, distinguishable by ${ }^{1} \mathrm{H}$ NMR and GC-MS $(\mathrm{dr}=50: 40)$; 13a: $(32 \%) ;{ }^{1} \mathrm{H}$ NMR $(400.13$ MHz) $\delta \quad 0.98(\mathrm{~d}, J=6.5 \mathrm{~Hz}, 3 \mathrm{H}), 1.30(\mathrm{~s}, 6 \mathrm{H}), 1.35-1.80(\mathrm{~m}, 12 \mathrm{H}), 3.97$ (s, $2 \mathrm{H})$; GC-MS $(70 \mathrm{eV}) \mathrm{m} / \mathrm{z}(\%) 237\left(\mathrm{M}^{+}, 2\right), 222(8), 194$ (38), 138 (79), 95 (100). 13c: (26\%); ${ }^{1} \mathrm{H}$ NMR (400.13 MHz) $\delta 0.87(\mathrm{~d}, J=6.4 \mathrm{~Hz}, 3 \mathrm{H}), 1.30(\mathrm{~s}, 6 \mathrm{H}), 1.35-1.80(\mathrm{~m}, 12 \mathrm{H}), 3.98$ (s, $2 \mathrm{H})$; GC-MS $(70 \mathrm{eV}) \mathrm{m} / \mathrm{z}(\%) 237\left(\mathrm{M}^{+}, 3\right), 222(9), 194$ (42), $138(88), 95$ (100). ${ }^{13} \mathrm{C}$ NMR and IR were measured on the mixture; ${ }^{13} \mathrm{C}$ NMR $(100.62 \mathrm{MHz}) \delta 15.9,16.0,21.6,21.7,22.7,22.8,27.6,27.7$, 28.4, 29.0, 29.7, 29.9, 33.4, 37.0, 37.7, 60.1, 60.2, 65.8, 65.9, 66.8, 66.9, 78.0, 78.6, 163.6, 163.7; IR (film) $v(\sim)$ 2920, 2860, 1660, 1520, 1450, $1380 \mathrm{~cm}^{-1} . \mathbf{1 3 b}$ and 13d were isolated in traces and identified by GC-MS.; 13b: GC-MS (70 eV) m/z (\%) $237\left(\mathrm{M}^{+}, 1\right), 222$ (4), 194 (15), 138 (70), 95 (100). 13d: GC-MS (70 eV) m/z (\%) 237 (M+1 1), 222 (5), 194 (20), 138 (85), 95 (100).

4,4-Dimethyl-2-(2,4-dimethyl-1-oxa-spiro[2.5]oct-2-yl)-4,5-dihydro-oxazole (14a, 14b). Overall yield $379 \mathrm{mg}$ (80\%). 4a: $265 \mathrm{mg}$ (56\%), yellow oil; ${ }^{1} \mathrm{H}$ NMR (400.13 MHz) $\delta 1.11$ (d, $\left.J=6.7 \mathrm{~Hz}, 3 \mathrm{H}\right), 1.29$ $(\mathrm{s}, 3 \mathrm{H}), 1.30(\mathrm{~s}, 3 \mathrm{H}), 1.32-1.80(\mathrm{~m}, 12 \mathrm{H}), 3.98(\mathrm{~s}, 2 \mathrm{H}) ;{ }^{13} \mathrm{C}$ NMR $(100.62 \mathrm{MHz}) \delta 14.0,17.0$, 19.1, 24.7, 25.5, 28.0, 28.1, 30.7, 32.5, 67.4, 69.5, 79.1, 164.5; GC-MS (70 eV) m/z (\%) 237 $\left(\mathrm{M}^{+}, 4\right), 222$ (5), 194 (30), 138 (65), 95 (100); IR (film) $v(\sim)$ 2920, 2860, 1660, 1520, 1450, $1380 \mathrm{~cm}^{-1} .4 \mathbf{b}: 114 \mathrm{mg}(24 \%)$, yellow oil; ${ }^{1} \mathrm{H}$ NMR (400.13 MHz) $\delta 1.12(\mathrm{~d}, J=7.0 \mathrm{~Hz}, 3 \mathrm{H})$, $1.28(\mathrm{~s}, 3 \mathrm{H}), 1.29(\mathrm{~s}, 3 \mathrm{H}), 1.35-1.80(\mathrm{~m}, 12 \mathrm{H}), 3.97(\mathrm{~s}, 2 \mathrm{H}) ;{ }^{13} \mathrm{C}$ NMR $(100.62 \mathrm{MHz}) \delta 13.7$, 16.6, 19.1, 25.1, 25.7, 28.1, 30.9, 32.5, 61.5, 69.3, 79.1, 164.4; GC-MS (70 eV) m/z (\%) 237 (M+, 4), 222 (6), 194 (31), 138 (69), 95 (100); IR (film) v( ) 2920, 2860, 1660, 1520, 1450, $1380 \mathrm{~cm}^{-1}$. 


\section{Acknowledgements}

This work was carried out within the framework of the National Project "Stereoselezione in Sintesi Organica: Metodologie ed Applicazioni" supported by the Ministero dell'Istruzione, dell'Università e della Ricerca, Rome. We also thank the University of Lecce for financial support.

\section{References and Notes}

1. Florio, S.; Troisi, L. Tetrahedron Lett. 1992, 33, 7953.

2. Bona, F.; De Vitis, L.; Florio, S.; Ronzini, L.; Troisi, L. Tetrahedron 2003, 59, 1381, and references therein cited.

3. Rao, A. S.; Paknikan, S. K.; Kirtane, J. G. Tetrahedron 1983, 39, 2323.

4. Gorzynski-Smith, J. Synthesis 1984, 629.

5. Dondoni, A.; Perrone, D. Aldrichimica Acta 1997, 30, 35.

6. Florio, S.; Capriati, V.; Luisi, R.; Abbotto, A.; Pippel, D. J. Tetrahedron 2001, 57, 6775.

7. Abbotto, A.; Capriati, V.; Degennaro, L.; Florio, S.; Luisi, R.; Pierrot, M.; Salomone, A. J. Org. Chem. 2001, 66, 3049.

8. Capriati, V.; Favia, R.; Florio S.; Luisi, R. ARKIVOC 2003, (xiv), 77.

9. De Vitis, L.; Florio, S.; Granito, C.; Ronzini, L.; Troisi, L.; Capriati, V.; Luisi, R.; and Pilati, T. Tetrahedron 2004, 60 (5), 1175.

10. Florio, S.; Troisi, L.; Capriati, V. J. Org. Chem. 1995, 60, 2279.

11. CCDC 229663 contain the supplementary crystallographic data for compound (+)-2a. These data can be obtained free of charge via www.ccdc.cam.ac.uk/conts/retrieving.html (or from the Cambridge Crystallographic Data Centre, 12, Union Road, Cambridge CB2 1EZ, UK; fax: +44-1223-336-033; or deposit@,ccdc.cam.ac.uk).

12. Suffert, J. J. Org. Chem. 1989, 54, 509.

13. Baldassarre, F.; Bertoni, G.; Chiappe, C.; Marioni, F. Journal of Molecular Catalysis B: Enzymatic 2000, 11, 55.

14. Djerassi, D.; Engle, R. R.; Bowers, A. J. Org. Chem. 1956, 21, 1547.

15. Chikashita, H.; Ikegami, S.; Okumura, T.; Itoh, K. Synthesis 1986, 5, 375.

16. Breton, P.; André-Barrès, C.; Langlois, Y. Synth Comm. 1992, 22, 2543.

17. Florio, S.; Capriati, V.; Luisi, R. Tetrahedron Lett. 1996, 37, 4781.

18. $t$-BuOCl could be conveniently prepared as described in: Mintz, M. J.; Walling, C. Org. Synth. Collect. 1973, 5, 184. 